\title{
A case of eosinophilic chronic rhinosinusitis associated with optic neuropathy
}

This article was published in the following Dove Press journal:

Clinical Ophthalmology

22 June 2011

Number of times this article has been viewed

\section{Takuji Kurimoto \\ Masahiro Tonari \\ Norihiko Ishizaki \\ Junko Matsuo \\ Hidehiro Oku \\ Jun Sugasawa \\ Tsunehiko Ikeda}

Department of Ophthalmology, Osaka Medical College, Osaka, Japan

Correspondence: Takuji Kurimoto

Department of Ophthalmology,

Osaka Medical College,

2-7 Daigaku-machi,

Takatsuki Osaka 569-8686, Japan

$\mathrm{Tel}+8 \mid 72683$ ।22।

Fax $+8|7268| 8 \mid 95$

Email kuritakul20II20I@yahoo.co.jp
Abstract: We report a case of eosinophilic chronic rhinosinusitis (ECRS) associated with optic neuropathy. The visual acuity in the right eye was suddenly reduced to no light perception on awakening in the morning. Fundus examination of both eyes on the same day showed no remarkable changes. Emergency computed tomography showed pan-sinusitis bilaterally and a partial defect of the sphenoid bone on the right side. From the clinical findings, the case was diagnosed as optic neuropathy associated with chronic sinusitis. Endoscopic sinus surgery (ESS) was performed on the same day, and all of the major sinuses were found to be filled with highly viscous fluid. Part of the optic canal had a defect probably due to inflammatory invasion from the adjacent sphenoid bone. Steroid therapy was started immediately postoperatively. Histopathological examination of excised polyps showed that numerous eosinophils had invaded the polyps but no hyphae were present. The patient reported that he had bronchial asthma and had had nasal polypectomy. Six months after the ESS and steroid therapy, the patient had a recurrence of the sinusitis. At that time, laboratory examination showed an elevation of total $\operatorname{IgE}$ and eosinophil numbers. From the clinical findings and course, this case was diagnosed as ECRS accompanied by optic neuropathy. Although ECRS rarely has ocular complications, the inflammation can spread and the optic nerve can be affected. Keywords: optic neuropathy, eosinophilic chronic rhinosinusitis, chronic sinusitis, allergic fungal rhinosinusitis

\section{Introduction}

Eosinophilic chronic rhinosinusitis (ECRS) has been established as a subtype of chronic rhinosinusitis (CRS). It is characterized by a resistance to conventional treatment with long-term macrolides, and frequent recurrences develop unless treated by steroids. ${ }^{1-3}$ ECRS is common in Asians. To make a diagnosis, the following must be examined: history of respiratory diseases; imaging tests of the rhinosinuses; laboratory data related to allergic reactions; and histopathological tests. ${ }^{3,4}$ Only one case of ECRS has been published that was associated with optic neuropathy, although several cases with optic nerve complications in cases of allergic fungal sinusitis have been reported. ${ }^{5,6}$

We have examined a case of ECRS with optic neuropathy probably caused by compression or inflammatory invasion of the optic canal by an expansion of the inflamed tissues. Even though endoscopic surgery (ESS) and steroid therapy were performed on the same day, the vision did not return.

\section{Case report}

A-68-year-old man noticed a sudden loss of vision in his right eye when he woke up. He was examined in our hospital the same day and his best-corrected visual acuity 
(BCVA) was no light perception in the right eye and 1.2 in the left eye. Color perception tests could not be performed on the right eye. The pupillary light reflex was almost absent in the right eye, and a relative afferent pupillary defect was present. Fundus examination showed no optic disc edema and atrophy, and spontaneous venous pulsations were not present. The macular area was normal, and optociliary shunt vessels were not present.

From the clinical findings, we suspected a retrobulbar lesion and ordered an orbital computed tomography (CT) on the same day. The CT scan showed a nasal obstruction. His history included nasal polypectomy more than 20 years earlier, and asthma that was not being treated. The CT scan also showed pan-sinusitis, multilobular nasal polyps, and a defect of the sphenoid bone (Figures $1 \mathrm{~A}-\mathrm{C}$ ). The right optic nerve appeared to be compressed by inflammatory tissues. Thus, we diagnosed the case as optic neuropathy due to extensive chronic rhinosinusitis. The laboratory data showed no elevation of the white blood cell count, eosinophilia, and $\mathrm{C}$ reactive protein.

We consulted an otorhinolaryngologist on the same day, and an emergency endoscopic sinus surgery (ESS) was performed. Intraoperatively, the ethmoid, maxillary, and sphenoidal sinuses were filled with highly viscous mucosal fluid. In addition, several polyps were found in
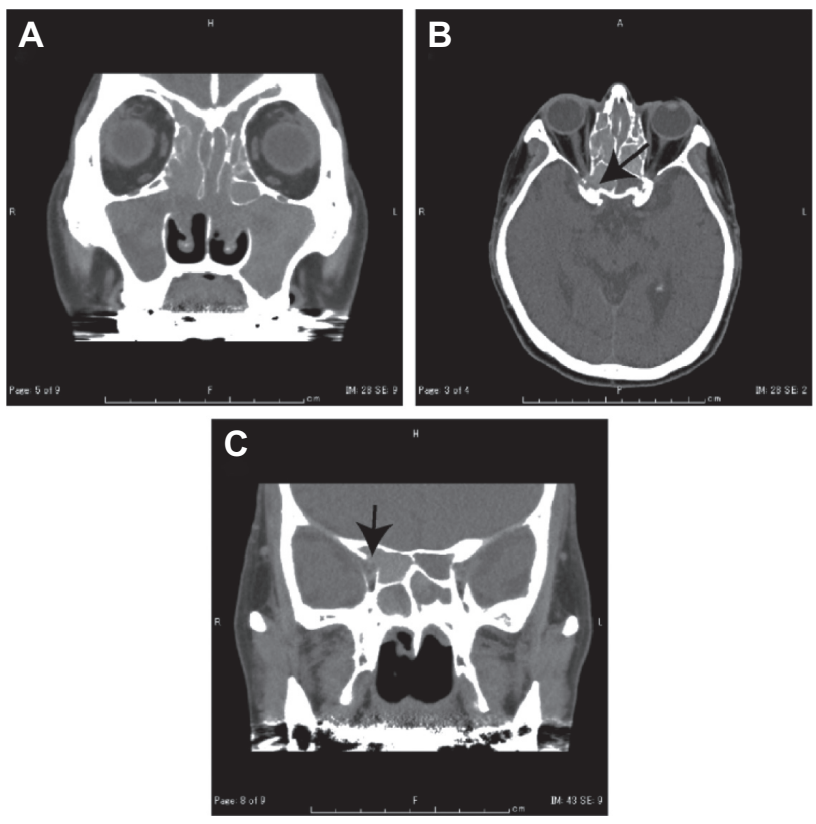

Figure I Computed tomographic (CT) images. Opacifications are present in the frontal, maxillary, anterior and posterior ethmoid, and sphenoid sinuses on both sides. A part of sphenoid bone is defective (arrows in B and C). (A) Coronal image showing opacity in the maxillary, ethmoid, and frontal sinuses. (B) Axial image. (C) Coronal image showing opacity present in the entire sphenoid sinus. A defect is also present in the sphenoid bone. the nasal cavity. After excision of the polyps and thickened mucosa and drainage of the mucosal fluid, an erosion and thinning of the lateral wall of the sphenoid sinus bone and partial defects of the optic canal were found. The optic canal was cleared to try to decompress the optic nerve, and betamethasone was applied to the exposed optic nerve to try to suppress the inflammatory changes.

Histopathological examination of the excised polyps showed an invasion of many eosinophils. No fungal hyphae were detected by Grocott and Periodic Acid Schiff (PAS) staining (Figures 2A-D). Unfortunately, the right visual acuity did not improve and remained at no light perception in spite of the postoperative betamethasone (total $324 \mathrm{mg}$ ). The fundus did not show any remarkable changes after the ESS and steroid therapy.

Six months later, nasal polyps recurred and nasal administration of betamethasone was started and is currently being continued. The laboratory data showed an elevation of total $\operatorname{IgE}$ and eosinophils compared to that before the surgery (before $1.2 \%$ and post-surgery 6.2\%). The specific IgEs against Candida and Aspergillus were negative with the radioallergosorbent (RAST) test.

\section{Comments}

Chronic rhinosinusitis (CRS) is a chronic inflammation of the rhinosinus mucosa and is characterized by the presence of two or more symptoms, viz., nasal discharge, nasal obstruction, post-nasal drip, and reduction or loss of the sense of smell. The diagnosis of CRS is made by the signs and symptoms
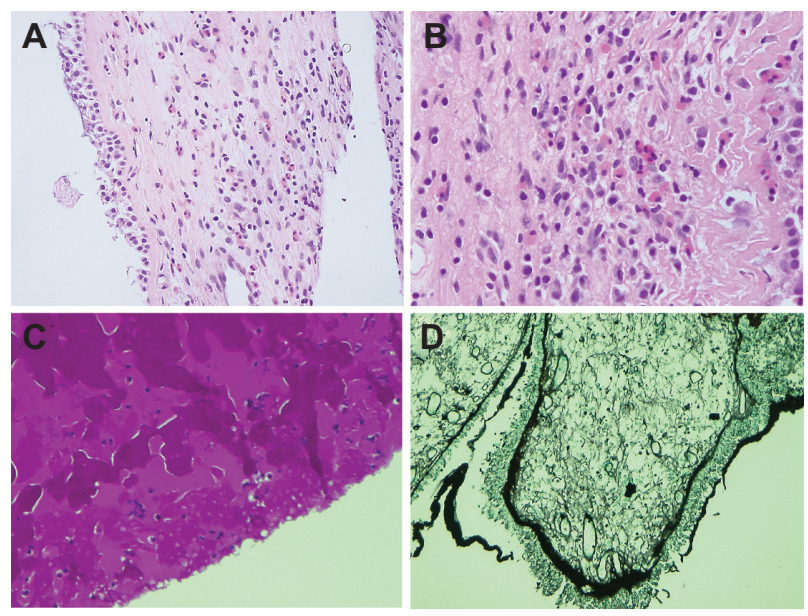

Figure 2 Photomicrographs of sections obtained from nasal polyp and mucosa. (A) and (B) Hematoxylin-eosin stained sections. Magnifications are $\times 200$ (A) and $\times 400$ (B). (C) PAS staining. (D) Grocott staining. Many eosinophils have invaded the subepithelium in the nasal polyp and are sporadically detected in the mucosal fluid. There are no hyphae in the polyps with Grocott and PAS staining. Abbreviation: PAS, Periodic Acid Schiff. 
that remain for longer than 3 months and opacification of the sinus in X-ray and CT images. Although CRS is common, its subclassification, pathology, and treatment have still not been completely established.

Cases of CRS have been divided into two groups; chronic rhinosinusitis with nasal polyps (CRSwNPs) and chronic rhinosinusitis without polyps (CRSsNPs) in Europe and the United States. CRSwNPs has a strong predisposition to recurrences even after ESS, and pathological examinations show numerous eosinophils invading the mucosa. In contrast, CRSsNP is diagnosed by purulent changes, and histopathological examinations of the nasal polyps and nasal mucosa show lymphocytes and neutrophils as the dominant inflammatory invaders. However, some cases of CRSwNP in Asians do not show eosinophil-dominant changes in the inflammation. Thus, other criteria are necessary to classify the cases. In addition, this subtype is not fully resolved by conventional long-term macrolide therapy combined with surgical dissection of the mucosal membrane in the sinuses. These cases have recently been classified as ECRS because they have different clinical characteristics from the more common CRSs that are associated with asthma, elevation of eosinophilic counts in peripheral blood, and massive invasion of eosinophils into the nasal mucosa.

The diagnosis of ECRS is finally determined by the clinical findings of chronic nasal symptoms, results of imaging tests, peripheral blood tests, and histological tests. ${ }^{4}$ Our case had the nasal symptoms, history of nasal polypectomy, and asthma. CT showed pan-sinusitis, and histopathological examination of removed mucosal membranes showed intensive invasion of eosinophils. Furthermore, there was a recurrence after surgery and steroid therapy. From the findings and clinical course, our case was diagnosed as ECRS and not the purulent type of CRS.

Unlike the purulent type of CRS, the ocular complications of ECRS have not been fully determined. Garg et al reported a case of ECRS associated with optic neuropathy. ${ }^{5}$ The MRI images showed a high intensity area over the left intraorbital optic nerve without any lesions in the paranasal sinuses. They treated their case with steroid therapy and radical sphenoethmoid clearance, however the visual acuity, which was light perception before treatment, improved only slightly to finger counting.

Fungi have received much attention in the United States as candidates for causing ECRS and allergic fungal rhinosinusitis (AFS). The diagnostic criteria for AFS include type 1 hypersensitivity to fungi, nasal polyps, and eosinophilic mucin containing fungi. ${ }^{7}$ It was suggested that fungi in the sinonasal mucosa might be the cause of non-atopic eosinophilia or local IgE production. ${ }^{8}$

The clinical findings of AFS overlap those of ECRS, and thus it is necessary to differentiate AFS from ECRS. Generally, cases with AFS have unilateral lesions, elevation of eosinophils, and $\operatorname{IgE}$ specific to fungal antigen. The important findings for diagnosis of AFS are the detection of hyphal forms of fungi and positive histopathological findings with PAS or Grocott staining. As with ECRS, AFS is rarely associated with optic neuropathy. ${ }^{6}$ The orbital findings of AFS are proptosis, ophthalmoplegia, optic neuropathy, and telecanthus. Imaging tests show hyper-attenuating foci along with expansion and destruction of the sinus walls. Most cases of optic neuropathy have an erosion of the lateral wall of the sphenoid and optic canal. ${ }^{6}$ The incidence of bony erosion in AFS is more than 12 times greater than in non-AFS. ${ }^{9}$ Whether an erosion of sphenoid bone occurs may determine the visual prognosis.

There is potential for mistaking the size of the lesion with only MRI. ${ }^{10}$ MRI of cases with allergic Aspergillus sinusitis showed that the lesions were low intensity cystic lesions in both T1 and T2 weighted images. Enhanced MR images show ring enhancement surrounding the low intensity areas. Thus, the lesions may appear to be smaller than the actual size unless enhanced MRI is used. By contrast, CT can accurately detect the area of hyper-attenuation and bone architecture.

Our case progressed to bilateral lesions, and the laboratory data were negative for IgE specific to fungi. In addition, there were no hyphae in the biopsy sections with PAS and Grocott staining. From these findings, AFS could be excluded from the differential diagnosis of our case.

It was not definitively determined why the visual acuity did not recover even though the ESS was performed within 30 hours after the initial symptoms of optic neuropathy developed. Our case presented with pan-sinusitis and thick mucosal membrane overall of the rhinosinus including the middle meatus and osteomeatal complex. Although the inflammatory changes gradually enlarged after the polypectomy, the drainage from the parasinus might have been blocked leading to infection and inflammatory changes. These changes could then cause the internal pressure in the parasinus to increase and affect the optic canal, even though bacterial infectious changes were not detected. In addition, local ischemia of the optic nerve might also have occurred secondary to the erosion of the optic canal followed by swelling of the optic nerve because the visual loss was so rapid. An earlier case of ECRS with optic neuropathy had a different 
MRI performed and an enhancement of the intraorbital optic nerve without extensive opacification of the rhinosinus was found. Although we did not do MRI, direct invasion by inflammatory tissues might have occurred in the right optic nerve as seen in the previous case.

In conclusion, we documented a rare case of ECRS complicated by optic neuropathy. Unfortunately, the visual acuity did not recover at all even though emergency ESS and steroid therapy were performed. In addition, a relapse of the nasal symptoms and signs occurred. These findings indicate that it is very important to suppress the allergic reaction for the prevention of ocular complications in ECRS cases.

\section{Acknowledgment}

We thank Professor Duco Hamasaki, Bascom Palmer Eye Institute, University of Miami School of Medicine, for editing this manuscript.

\section{Disclosure}

The authors report no conflicts of interest in this work.

\section{References}

1. Ishitoya J, Sakuma Y, Tsukuda M. Eosinophilic chronic rhinosinusitis in Japan. Allergol Int. 2010;59(3):239-245.

2. Takeno S, Hirakawa K, Ishino T. Pathological mechanisms and clinical features of eosinophilic chronic rhinosinusitis in the Japanese population. Allergol Int. 2010;59(3):247-256.

3. Sakuma Y, Ishitoya J, Komatsu M, et al. New clinical diagnostic criteria for eosinophilic chronic rhinosinusitis. Auris, Nasus, Larynx. 2011; 38(5):583-588.

4. Matsuwaki Y, Ookushi T, Asaka D, et al. Chronic rhinosinusitis: risk factors for the recurrence of chronic rhinosinusitis based on 5-year follow-up after endoscopic sinus surgery. Int Arch Allergy Immunol. 2008;146 Suppl 1:77-81.

5. Garg A, Das-Bhaumik R, Nesbitt AD, et al. Visual loss secondary to eosinophilic mucin rhinosinusitis in a woman: a case report. $J$ Med Case Reports. 2010;4:350.

6. Thakar A, Lal P, Dhiwakar M, Bahadur S. Optic nerve compression in allergic fungal sinusitis. $J$ Laryngol Otol. 2011;125(4):381-385.

7. Sasama J, Sherris DA, Shin SH, Kephart GM, Kern EB, Ponikau JU. New paradigm for the roles of fungi and eosinophils in chronic rhinosinusitis. Curr Opin Otolaryngol Head Neck Surg. 2005;13(1):2-8.

8. Sheahan P, Ahn CN, Harvey RJ, et al. Local IgE production in nonatopic nasal polyposis. J Otolaryngol Head Neck Surg. 2010;39(1):45-51.

9. Ghegan MD, Lee FS, Schlosser RJ. Incidence of skull base and orbital erosion in allergic fungal rhinosinusitis (AFRS) and non-AFRS. Otolaryngol Head Neck Surg. 2006;134(4):592-595.

10. Demaerel P, Brown P, Kendall BE, Revesz T, Plant G. Case report: allergic aspergillosis of the sphenoid sinus: pitfall on MRI. Br J Radiol. 1993;66(783):260-263.
Clinical Ophthalmology

\section{Publish your work in this journal}

Clinical Ophthalmology is an international, peer-reviewed journal covering all subspecialties within ophthalmology. Key topics include: Optometry; Visual science; Pharmacology and drug therapy in eye diseases; Basic Sciences; Primary and Secondary eye care; Patient Safety and Quality of Care Improvements. This journal is indexed on

\section{Dovepress}

PubMed Central and CAS, and is the official journal of The Society of Clinical Ophthalmology (SCO). The manuscript management system is completely online and includes a very quick and fair peer-review system, which is all easy to use. Visit http://www.dovepress.com/ testimonials.php to read real quotes from published authors. 